\title{
The Narrative Logic of Rube Goldberg Machines
}

\author{
David Olsen ${ }^{1}$ and Mark J. Nelson ${ }^{2}$ \\ 1 Aeiouy Computer Media \& Entertainment, Hollister, CA, USA \\ dolsen@aeiouy.org \\ 2 The MetaMakers Institute, Falmouth University, Cornwall, UK \\ mjn@anadrome.org
}

\begin{abstract}
Rube Goldberg's cartoons famously depict absurd, unreasonably complex machines invented by Professor Lucifer G. Butts to carry out simple tasks. Rube Goldberg machine has now become a byword for overly complicated machinery or bureaucracy of any kind. The specific structure of Goldberg's original cartoons, however, is quite interesting. Beyond simply being complex, his machines are based on a particular repertoire of objects used in stereotypical, coincidental, and comical ways, exhibiting almost as much of a narrative logic as a mechanical logic. In this paper, we analyze the structure of these cartoon machines' construction, with a view towards being able to generate them using a planning formalization of this analysis.
\end{abstract}

Keywords: Rube Goldberg machine, computational humor, narrative generation, planning

\section{Introduction}

One of the signature gags of cartoonist Rube Goldberg (1883-1970) was to "invent" fantastical, complex machines that, through a series of absurdly unnecessary intermediary operations, would eventually succeed in performing a simple task. This genre is now termed the Rube Goldberg machine [3,5,10], and a few examples are shown in Figures 1-3.

We put "invent" here in scare quotes not only because of the machines' obvious lack of practical utility for accomplishing their stated goals, but because the original machines as conceived by Goldberg himself exist only in a cartoon world, not as physical machines. The concept has since inspired people to build complex real-world contraptions that capture some of these machines' spirit, and which are also termed Rube Goldberg machines. The real-world machines, however, exhibit a quite different logic, one more akin to an extended version of a domino show than to the absurdist logic of Goldberg's cartoons (as we will discuss further).

This paper's main aim is to structurally analyze the logic followed by Rube Goldberg machines in their original cartoon-world formulation. Goldberg intended these impractical inventions in part as "symbols of man's capacity for exerting maximum effort to accomplish minimum results" [10, p. 53]. But beyond 


\section{A Simple Way to Take Your Own Picture}

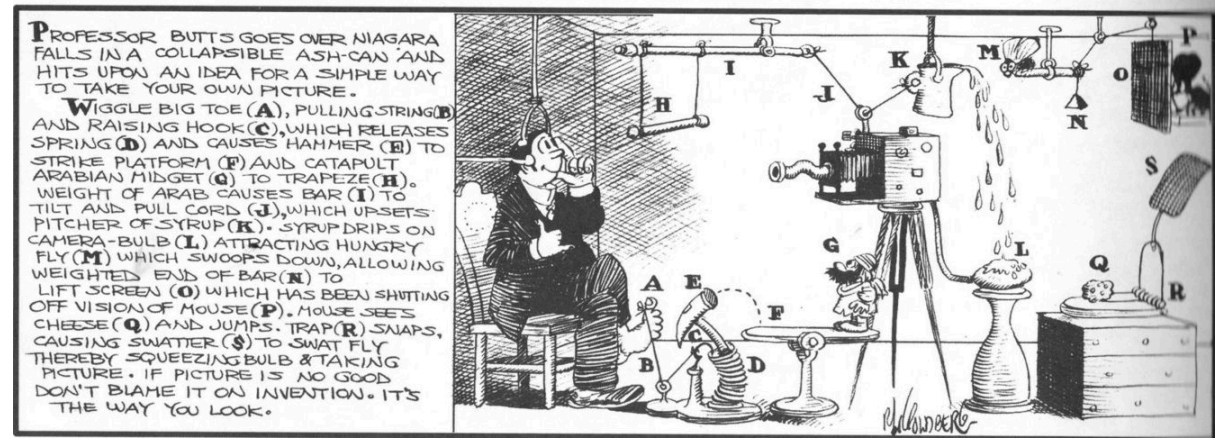

Fig. 1. A Simple Way To Take Your Own Picture

the unnecessary mechanical complexity, the machines gain additional absurdity and impracticality by incorporating components that would be unlikely to work reliably (or at all) in a real machine, such as the actions and reactions of animal characters. In addition, many of the machines exhibit violence in their operation, towards both animals and humans, that makes them uncomfortable to contemplate - and unethical to build - as real machines.

Based on our analysis of the logic of Rube Goldberg machines, we have implemented a preliminary generator, in a planning formalism, to produce novel machines in this style. This implementation is intended in part as a generative test of our analysis, and in part as our actual practical medium-term goal. Besides being interesting to analyze, our focus on Rube Goldberg machines comes from a desire to procedurally generate interactive videogame puzzles in this style. Videogame puzzles have previously been generated using a simple backwardschaining planner [1], which is an approach we also adopt here. But here our goal is to set up the planning problem so that the resulting puzzles have the characteristically comical Rube-Goldberg-machine feel.

\section{Cartoon and Physical Machines}

The general concept of a Rube Goldberg machine is culturally widespread, to the point where it has become a byword for any overly complicated apparatus or process, from physical devices to bureaucratic procedures. Goldberg's machines themselves, however, have a much more specific character beyond the basic starting point of being unnecessarily complicated, which it is our goal to model.

Goldberg's machines are always in cartoon form, and almost none could actually be built, for reasons ranging from their dependence on coincidence to their incorporation of animal cruelty (as will become apparent from the explanation of a few machines in the following section). In this absurd form of mechanization 
they bear some similarity to roughly contemporaneous depictions of machines in avant-garde art, especially by the Dadaists, who appear to have been aware of Goldberg's work, although he himself was uninterested in "high art" and didn't comment on theirs [4].

People have however built physical machines often described as Rube Goldberg machines, and it is worth briefly looking at how they differ. To focus on an example that also has a significant narrative running through it (rather than being purely a complex machine), the Japanese television show Pythagora Switch has built a series of strongly narrative-driven domino-like machines described as Rube Goldberg machines [9]. These have also become popular in the west after being uploaded to YouTube with English subtitles. ${ }^{3}$ In these machines, a character, Biisuke, engages in adventures, as he is captured at one point, rescued by friends, etc., all through the operation of an intricate series of chained mechanical reactions.

These mechanical reactions essentially form a large domino show, but with more diverse objects than simply falling dominoes. The logic of operation is that each movement hits the next device, triggering it to move and continue the sequence. Therefore the design is largely based around space: the machine components must all physically chain with each other, and there are generally recurring elements, such as in the case of the Biisuke machines a track for rolling balls, that are re-used to connect parts together.

In cartoon Rube Goldberg machines, by contrast, space is not really the primary concern. In a sense they are actually much simpler. There is a goal state, and the intervening steps are mostly there to be funny, not to contribute to an impressive feat of virtuosic machine-building, as in the physical examples. The only real requirement on the components in a cartoon machine is that each plausibly connects the chain, leaning heavily on the reader being able to implicitly read what its role in the machine is.

It is usually conceptually clear to the reader what role each of the components play in the cartoon machines, but their actual function depends on coincidence, luck, and suspension of disbelief. The overall machine does thus exhibit a kind of mechanical logic in which the apparatus achieves an end goal through a sequence of internal events chained together in a logically consistent manner. But it is an absurdist mechanical logic, which incorporates characters - in most cases characters whose presence is not even really necessary in the first place - who react to events and to other characters.

Therefore in some respects the logic of (cartoon) Rube Goldberg machines is more similar to the internal logic of narrative structures, which require some degree of coherence but not strict fidelity to physics, than it is to the strictly mechanistic logic of real-world machines. Our attempt here to discern a kind of coherent narrative logic in Rube Goldberg machines is therefore related to the (relatively sparse) existing work on investigating the internal logic of cartoons, such as the Road Runner and Coyote scenarios of Looney Tunes [6, 8].

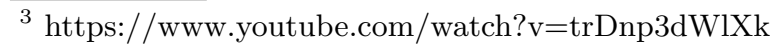




\section{Taking the Shirt off the Taxpayer's Back}

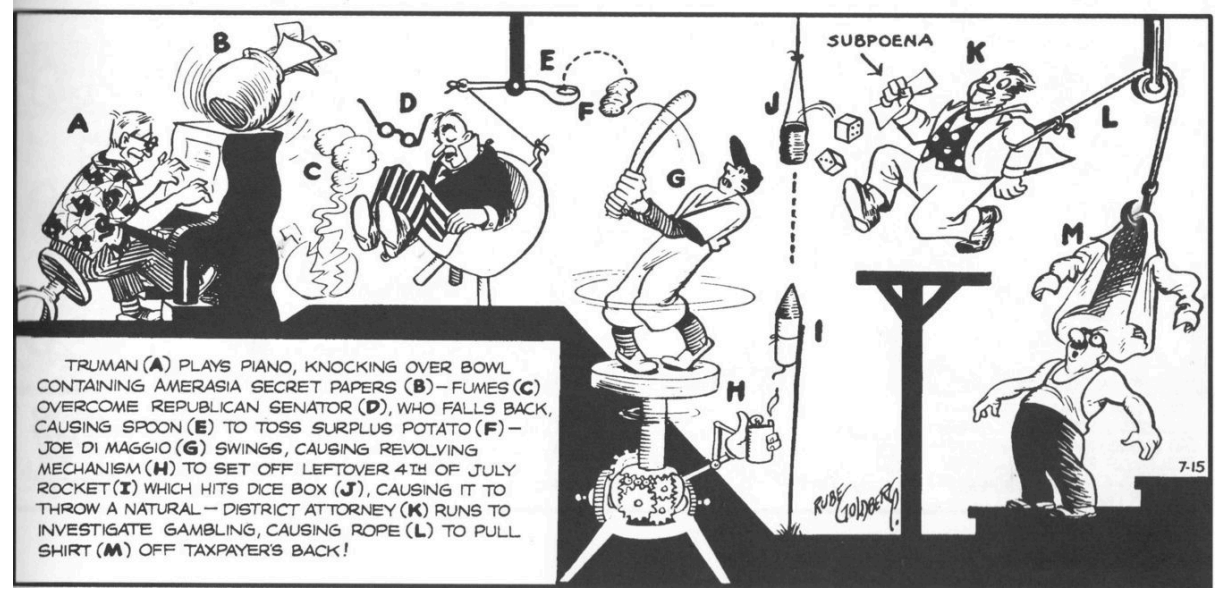

Fig. 2. Taking the Shirt off the Taxpayer's Back

\section{Principles of Rube Goldberg Machines}

A single Rube Goldberg invention is a comic with three separate parts: the title, the cartoon, and the description. The title could be considered the most important part for interpretation, as it provides a framework for the action in the world, explaining the practical goal that the invention is trying to accomplish. The cartoon depicts the placement of objects in the world, each labeled. Finally the text describes what is going on, i.e. the potential moves that link the objects labeled together to achieve the stated goal.

The machines themselves are invariably complex, often ridiculous and while always providing a path to the end goal described in the title, the end goal is something often beyond expectation. Besides being large and overly complex, they tend to twist the meaning behind what they do, subverting expectations either through the outcome, the components, or the reader-assumed motivations for the machine. What makes them more ridiculous is that despite having required apparent feats of ingenuity to invent such a complex machine, they can only be used once without rebuilding. The machines' initial condition is almost always a precarious arrangement of components, some even in mid-motion or in rather unlikely scenarios. If the machine were actually set in motion-which is never shown explicitly, only implied by the arrangement of components - all this careful design would come crashing down, with most components ending up out of place and some destroyed. Running the machine a second time would involve carefully setting up these unlikely scenarios that got the machine to work in the first place (after possibly sourcing new components).

Marzio [5, p. 145] reports that Goldberg drew at least one of these machines per week between 1909 and 1935, amounting to over 1,000 examples, although to our knowledge there is no comprehensive archive available, and only a small 
subset are collected in anthologies. In this section we break down the common types of components of Rube Goldberg machines, as well as what role those components play in the overall machines' operation and narrative, based primarily on analyzing the subset of machines collected in an anthology published in 2000 [10].

\subsection{Chaining components}

Despite all the complexity when the viewer looks at the machines, with steps that are numerous, ridiculous, and unlikely, it should nonetheless be immediately apparent that the machines have a potential to work. Each thing placed in the world provides the necessary conditions for that particular component to work, forming a chain of coincidental action that produces a result, even if not exactly the one advertised. The machines can be made of humans, animals, or inanimate objects, most often some sort of mixture.

Each step may seem contrived or even random, but it fits with its context: the item earlier in the chain must cause an action that this item uses, in a sense, to complete its own action, which then leads to the next item down the chain. In some of Goldberg's own discussions, this can sound like a machine constructed through backwards-chaining planning:

"For instance, when I have a goat crying in one of my cartoons, I have to give a satisfactory reason for having him cry. So I have someone take a tin can away from him." — Rube Goldberg [7]

The steps are thus pairs of objects with actions gluing them together; the state of one object and action must fit with what the next object needs.

\subsection{Beginning, middle, and end}

Objects can be roughly divided into three regions of the machine: The initial object, the final object, and the ones in between. The initial object uses a predetermined state of the world to start the action. This object itself may even be reasonable by the standards of the objects relating to the goal; for example, a mouse may simply be standing in the area (because it is later needed for shooting into space). This is not always the case though, and sometimes the initial choice may also seem whimsical or random.

The final object is the final step achieving the goal. It illustrates the purpose of the machine, but as with the rest of the machine, there may be twist expectations of what is supposed to be done to achieve the nominal goal of the machine. A machine to get rid of a mouse, for example, ends by shooting it into space. While this is legitimate way of removing a mouse, it is not the end goal - a dead mouse on earth - that we might expect.

The objects that make up the bulk of the machine, in the middle, are much less constrained, and often seem almost random in how they are picked. They can vary in type, but don't necessarily need to. They can vary in placement, 


\begin{tabular}{|c|c|c|c|}
\hline Category & Subcategory & Description & Example \\
\hline \multirow[t]{4}{*}{ Human } & Generic & $\begin{array}{l}\text { Person who does things a } \\
\text { stereotypical human would } \\
\text { likely do in a situation. }\end{array}$ & $\begin{array}{l}\text { A person at a lunch } \\
\text { table will probably } \\
\text { eat their sandwich. }\end{array}$ \\
\hline & Object & $\begin{array}{l}\text { Hybrid person/object } \\
\text { subsystem. Human is the } \\
\text { motive force, but only as engine } \\
\text { for movement. }\end{array}$ & $\begin{array}{l}\text { Person sitting on a } \\
\text { chair falling over. }\end{array}$ \\
\hline & Coincidence & $\begin{array}{l}\text { Human as an engine for } \\
\text { coincidence to occur. }\end{array}$ & $\begin{array}{l}\text { Person slips on } \\
\text { banana peel. }\end{array}$ \\
\hline & Attribute & $\begin{array}{l}\text { Person with a characteristic } \\
\text { that causes them to act in a } \\
\text { stereotypical way. }\end{array}$ & $\begin{array}{l}\text { Baseball player will } \\
\text { hit baseball thrown at } \\
\text { them in any situation. }\end{array}$ \\
\hline Animal & Attribute & $\begin{array}{l}\text { Attribute that is viewed to be } \\
\text { inherent in an animal. }\end{array}$ & $\begin{array}{l}\text { Mice are attracted to } \\
\text { cheese. }\end{array}$ \\
\hline \multirow[t]{3}{*}{ Item } & Subsystem & $\begin{array}{l}\text { Multiple items that combine to } \\
\text { create a single action. }\end{array}$ & $\begin{array}{l}\text { A pulley and rope for } \\
\text { lifting things. }\end{array}$ \\
\hline & Physics & $\begin{array}{l}\text { Item with a physical } \\
\text { characteristic that allows it to } \\
\text { move in the world. }\end{array}$ & $\begin{array}{l}\text { A feather floats up in } \\
\text { a puff of air. }\end{array}$ \\
\hline & Attribute & $\begin{array}{l}\text { Item with a characteristic } \\
\text { affecting animate objects. }\end{array}$ & $\begin{array}{l}\text { A piece of cheese } \\
\text { attracts a mouse. }\end{array}$ \\
\hline
\end{tabular}

Table 1. Types of objects commonly found in Rube Goldberg machines

but again that isn't necessary. The only real rule seems to be that when viewed through the lens of a single step, the action must seem somewhat reasonable (a very relative term in this case), as well as - and this is a hard requirement-look overly complex and ridiculous from a wider perspective.

\subsection{Types and roles of objects}

The types of objects commonly found in Rube Goldberg machines can be grouped based on certain attributes of the objects, or of actions that the objects can perform. Table 1 summarizes three primary types of objects: humans, animals, and (inanimate) items. Each type of object can be further categorized by which of the object's characteristics the machine wants to use, as follows.

Humans. The sub-categories that humans can be split into are: humans as a motive force, humans representing an occupation or other noteworthy characteristic, generic humans, and humans as actors in a coincidence. Humans as a motive force put people into the role of a human-looking machine; they can be attached to other inanimate components and provide the force needed to run those components. For example, a human can push a lever down, the same work that could have been done by a rock. Humans with an obvious occupation or 
other noteworthy characteristic can be used by the machine to imply an obvious action; these sometimes include characters with specifically identified names or titles. Joe DiMaggio in Figure 2 shows up swinging a bat, because when a baseball player sees a baseball and they have a bat, of course they will swing at it. When humans are used to act like generic humans, they act in stereotypical (though perhaps exaggerated) ways and often poke fun at some aspect of human nature. They do "normal" things: they answer phones, they pick interesting things off the ground, and all manner of other activities. Finally, humans can be a object taking part in a coincidence. They again are a motive force in this case, but an entity with movement whose sole reason for existence is because someone had to be there to slip on that banana peel.

Animals. Animals have no subcategories. In every case we have found, the role of an animal in a Rube Goldberg machine is the same: to embody a stereotypical attribute perceived to be inherent in that type of animal (similar to the subcategory of humans who are used to represent an occupation). Implied animal attributes usually result in particular actions that the animal is obviously supposed to take. Mice like cheese, so they will try to eat it; goats eat anything so they will chew on that tin can; bulls are ornery so they will charge at people, etc. These characteristics don't have to be particularly accurate, just something that popular conception has this type of animal doing that would be immediately apparent to most readers.

Items. Inanimate items can be split into one of several subcategories: multiple items that form a sort of subsystem, items with physical properties, or items with some sort of attribute that has an affect on a human or animal. The subsystem might be a pulley attached to the trigger of a gun on one end and a weight on the other. This whole apparatus is a single conceptual step in the process and therefore we consider it as one item. Objects used for their physical characteristics might be a shape, like a wedge, or some other more active characteristic, like being magnetic. This is generally related to movement of the object or other inanimate objects. Items with attributes that affect animate objects typically cause the human or animal to do something, but don't necessarily do anything themselves. A mouse is attracted to cheese, but all the cheese has to do is be in sight of the mouse; the action is on the part of the mouse.

The same object can serve, and often does, in more than one of the subcategories at different points during the machine's operation; each step may use a different characteristic of an object. For example, oranges have the physical characteristic of being round, so they can roll, but they also attract birds who want to eat them. So there might be a sequence such as: 1 . a bird flies to the orange to eat it, but instead pushes it down, 2. The orange rolls off the bench and hits something.

\subsection{Example}

To illustrate the principles of Rube Goldberg machines outlined in the previous section, we'll walk through an analysis of the example shown in Figure 3. The basic characteristic of the comic is typical of a Goldberg invention. Here the 


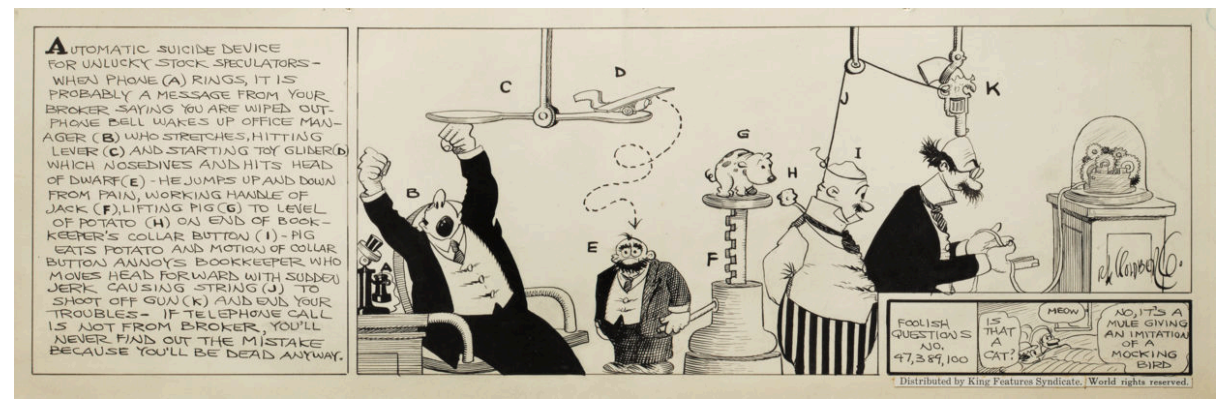

Fig. 3. Automatic Suicide Device for Unlucky Stock Speculators

textual description on the left side gives context to the comic on the right side, and the title states the overall goal. The point of the machine in this comic is rather morbid: it is a suicide machine for people who have received bad news about their stock portfolio.

The outside factor that starts the action is receiving a phone call (presumably from a stock broker). The action sequence from that point on is: the telephone wakes a sleeping office manager, who pushes a lever, which knocks over a glider, which lands on a dwarf, who jacks up a jack, which pushes up a pig, who eats a potato, which bothers a bookkeeper, who moves down, pulling a string, which pulls the trigger on a gun. Unpacking these events in detail:

1. Initial state. The telephone is ringing because it received a call (presumably from the stock broker). This is a physical characteristic of phones.

2. The telephone ringing wakes up the sleeping office manager. An attribute of phone ringing is that it can awaken sleeping people (or perhaps animals). Generic people are awakened by loud noises.

3. The office manager stretches when awakened, and in doing so hits a lever. This is a human providing motive force for an item. The item has the physical characteristic of being able to be pushed.

4. The lever drops the glider that was on the other side of the lever. This is a physical characteristic of one object causing the physical characteristic of another object to be activated.

5. The glider hits the dwarf in the head. The physical characteristic of the glider matches with human coincidence of happening to be under the falling glider.

6. The dwarf jumps up and down, causing the jack lever to move, pushing up the jack. The dwarf is acting as a generic human, jumping up and down while angry (as angry people are stereotypically depicted in cartoons). The jack has a physical characteristic that causes it to rise when used.

7. A pig who is on the jack eats the potato, because it is now within range. Pigs are animals who like to eat things - such as potatoes, which have an attribute of attracting pigs to eat them. 
8. However the potato is attached to the bookkeeper, so it causes annoyance when eaten, which causes the bookkeeper to move. In this case it is a human/item system, and is caused by generic human characteristics (being annoyed, moving in response to annoyance) with the same human involved on both sides.

9. The bookkeeper is also attached to a string, which is part of a string/pulley system. The bookkeeper is part of another human/item system which is attached to an item subsystem.

10. The string is furthermore attached to a trigger on a gun, which is pulled, thereby shooting the gun. This is the item subsystem using physical characteristics of an object.

11. Final state. A dead person, shot.

The first thing to note is that the machine does set out what is supposed to do: the person is dead in the end. It twists expectations by causing the death not just because of a bad call from the stock broker, but if any call is received on this particular telephone.

The next thing to note is that one can see the biggest characteristics of Rube Goldberg machines: it is long and complicated to do something that would be quite easy to do otherwise. The only required items for this machine are the telephone, gun and person getting shot.

Given that it is a Rube Goldberg machine, we can see that actions of objects do have reasonable, if unlikely, reasons for happening. Why a bookkeeper has a potato on his collar, the viewer may never know. But it is reasonable to expect that a pig might eat the potato if it saw it there to be eaten, and this would understandably cause distress to a person who would then move, all reasonable given the circumstances.

The choice of objects placed for this particular machine are a mixed bag, some random and some suited to the setting. Most of the people don't seem out of place. Some of the items are also reasonable; having a telephone in an office is normal and would likely to be there even in a more realistic scenario, and the gun in an office is not too unreasonable - this is America, after all.

The rest is less clearly there for a reason. Why is there a pig? Why was there a potato attached to a collar? Could the pig have been replaced by a goat, and the potato with a tin can, and produced a similar result? How could a system be designed to generate this kind of scenario?

\section{Generating Rube Goldberg Machines}

From our analysis of Rube Goldberg machines' structure, the basic operation of a generator becomes clear. We represent object/action pairings as plan operators, and their various possible roles in a machine and relationships to other objects (stereotypical, physical, etc.) are encoded as operator preconditions and postconditions. The actual planning is quite straightforward, simply chaining together actions so that pre- and post-conditions match; the bulk of the logic that captures the nature of Rube Goldberg machines is in the design of the operators. 


\begin{tabular}{lll}
\hline & Precondition & Postcondition \\
\hline 1 & Cat sees mouse & Cat chases mouse \\
2 & Cat is chasing mouse & Mouse runs away \\
3 & Mouse runs over a button & A button is pressed \\
4 & A button is pressed & A light turns on \\
\hline
\end{tabular}

Table 2. Actions redesigned as pre-conditions and post-conditions

\subsection{Designing operators}

Consider the following four-step machine to turn on a light:

1. The cat chases the mouse.

2. The mouse runs towards the hole.

3. The mouse presses button (by running over it).

4. The button turns on the light.

Each of these steps must have a reason for it to occur, e.g. the mouse is running because a cat is chasing it. The objects that are causing the action to occur must then in turn be present and similarly motivated. Table 2 shows these four steps analyzed into precondition and postcondition pairs that relate an action done by one object to another.

We turn each precondition and postcondition pair here into a plan operator that matches actions and objects, and use a STRIPS-style planner [2] to chain them together in a coherent way. World state, and the pre/post-conditions that change it, generally represents either the presence of objects, or the recent occurrence of an action that might motivate another action. In contrast to standard STRIPS planning world state therefore does not stay constant until explicitly changed; state representing recent actions that might motivate a subsequent action "times out" if not used.

One big advantage when planning in Rube Goldberg machines is that we can always assume coincidence will help us achieve the outcome that is most advantageous to us, when multiple outcomes are possible. This allows us to define a minimal list of preconditions and postconditions, since we can always take the desired outcome, the one that might happen if we want it to.

In addition to regular state, there are a few pseudo-states that can be asserted and checked to influence the behavior of the planner. From our analysis of Rube Goldberg machines, there are often certain constraints on object reuse, or on placement of objects with similar postconditions. For example, a reason for the mouse running is the important part, and having more than one mouse will just confuse matters and make the machine's logic less apparent. To capture these constraints, no_repeat tells the system to not re-use an operator even if the preconditions match, and single_matched_state tells the system to not use a operator that has a postcondition which was already used elsewhere. This allows operators to influence some of the higher-level structure of the machines, limiting confounding elements that would confuse the main line of the action. 


\begin{tabular}{lll}
\hline Plan Conditions & single_matched_state & \\
\hline Initial State & $\begin{array}{l}\text { mouse_exists, cat_exists, mouse_seen, } \\
\text { cheese_exists }\end{array}$ & \\
\hline Operator & Preconditions & Postconditions \\
\hline cat_chasing & $\begin{array}{l}\text { cat_exists, mouse_exists, mouse_seen, } \\
\text { no_repeat } \\
\text { cat_exists, mouse_exists, cat_chasing, }\end{array}$ & cat_chasing \\
mouse_running & mo_repeat \\
cheese_sunning & \\
button_pressed & mouse_running & mouse_running \\
light_on & button_pressed & $\begin{array}{l}\text { button_pressed, } \\
\text { no_repeat }\end{array}$ \\
\hline Goal State & light_on & \\
\hline
\end{tabular}

Table 3. Example plan

\subsection{Example generated plan}

A simple planning problem using the operators abstracted from the four-step machine discussed above is shown in Table 3. Each of the operators shows a causal link between actions where the preconditions match the postconditions. We can use the fact that in a Rube Goldberg machine we don't have to worry about what could happen but rather what we want to happen. So the fact that the mouse runs over the button when it potentially could have run anywhere is the system's choice and not the result of any kind of "honest" simulation.

The group of actions shown here can produce two possible plans: cat_chasing, mouse_running, button_pressed; or, similar but with a different initial action, cheese_seen, mouse_running, button_pressed. These two plans just show the basic variety that can be generated from a few operators; longer, more complicated plans come naturally given more operators - although whether that alone is enough to make them sufficiently Rube Goldbergesque remains an open question, discussed below.

\section{Conclusions and Future Work}

This paper presents a structural analysis of Rube Goldberg machines, specifically of the narrative logic used in the machines depicted in Rube Goldberg's own cartoons. Besides the well-known unnecessary complexity of these machines"maximum effort to accomplish minimum results" - the cartoon machines usually include human or animal characters as machine components, and their implied operation heavily relies on readers being able to recognize stereotypical actions suited to, for example, mice or goats or stockbrokers. Often these machines would not actually work, based as they are on coincidences, but each 
component sufficiently matches those that come before and after it in the sequence that the reader can read what is supposed to happen. In that sense they have an internal narrative consistency.

We further extracted some of the more concrete principles used by Professor Lucifer G. Butts in designing his inventions. The inventions' most iconic feature is found in the types of components and how they are suggestively arranged without ever showing the machine explicitly operating. Machines can include human, animal, and inanimate objects, each of which is used in a specific set of ways, summarized in Table 1, that both effect the machine's operation and provide motivations for the existence and operation of the machines' other other components. Using this analysis, we built a planning engine that takes potential Rube Goldberg machine elements defined as plan operators, and chains them into plausible, coherent machines that follow the logic as we've analyzed it in this paper.

Future work involves fully realizing the generation of machines. We currently generate only half of a typical Rube Goldberg cartoon: the textual half that explains the chain of actions comprising the machine's operation. The other half, of course, is the drawn cartoon machines themselves. To generate that requires laying out the components spatially, with a suitable visual style and setting. The constraints for layout in a cartoon world based on coincidence are fairly loose, but nonetheless not entirely absent, and constraints on layout might even be able to drive aspects of the generation process. In addition, the title is often important, and we do not currently generate a title for the machine; or, what might be even better, generate a machine for a given title.

In addition, we have only a small number of operators in our current prototype, so cannot generate nearly the range of machines seen in Goldberg's works. We have slowly added to this by analyzing more original cartoons and identifying actions in them. However our current approach of individually handwritten operators representing specific object/action combinations does not scale very well; we would like to move towards a more factored representation, based on a dictionary of items with defined attributes from which actions fitting several types of schema can be generated. This would allow for automatically generated operators with preconditions and postconditions that still fit the typology of Rube Goldberg machine actions as we've analyzed it here.

Finally, our analysis here is structural, while many of the adjectives we've used to describe Rube Goldberg machines - "absurd", "comical", "ridiculous" seem to have a clearly stylistic component. It may be that some of these arise naturally by virtue of the structure, since a complex machine that depends on animal components and coincidence has a certain amount of built-in absurdity. But to produce interesting, coherent cartoons along the lines of those drawn by Goldberg, we may need a more explicit model of style, and/or an explicit model of narrative features such as absurdity. An explicit reader model may also be needed in order to generate machines where the narrative logic is, while absurd, still apparent; the "pseudo-states" used in Section 4.1 to ensure that the main line of action in the machine isn't obscured are an ad-hoc step in that direction. 


\section{References}

1. Dart, I., Nelson, M.J.: Smart terrain causality chains for adventure-game puzzle generation. In: Proceedings of the IEEE Conference on Computational Intelligence and Games. pp. 328-334 (2012)

2. Fikes, R., Nilsson, N.: STRIPS: A new approach to the application of theorem proving to problem solving. Artificial Intelligence 2(3-4), 189-208 (1971)

3. Goldberg, R., Keller, C.: The Best of Rube Goldberg. Prentice Hall (1979)

4. Marzio, P.C.: Art, technology and satire: The legacy of Rube Goldberg. Leonardo 5(4), 315-324 (1972)

5. Marzio, P.C.: Rube Goldberg: His Life and Work. Harper \& Row (1973)

6. McCartney, R., Anderson, M.: The believability of Road Runner cartoons: Logical consistency conquers unreality. In: Proceedings of the AAAI Workshop on Entertainment and AI / A-Life. pp. 6-11 (1996)

7. O'Connor, B.: Inside the whimsical but surprisingly dark world of Rube Goldberg machines. The Verge (April 2015), https://www.theverge.com/2015/4/22/8381963

8. Olsen, D., Mateas, M.: Beep! Beep! Boom!: Towards a planning model of Coyote and Road Runner cartoons. In: Proceedings of the 4th International Conference on Foundations of Digital Games. pp. 145-152 (2009)

9. Plante, C.: This adorable Rube Goldberg machine tells the story of brotherly love. The Verge (April 2017), https://www.theverge.com/2017/4/21/15382804

10. Wolfe, M.F., Goldberg, R.: Rube Goldberg: Inventions. Simon \& Schuster (2000) 